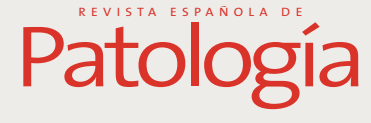

www.elsevier.es/patologia

REVISIÓN

\title{
Actualización de las recomendaciones para la determinación de biomarcadores en el carcinoma de pulmón avanzado de célula no pequeña. Consenso Nacional de la Sociedad Española de Anatomía Patológica y de la Sociedad Española de Oncología Médica
}

\author{
Fernando López-Ríos ${ }^{a, *}$, Javier de Castro ${ }^{b}$, Ángel Concha ${ }^{c}$, Pilar Garrido ${ }^{d}$, \\ Javier Gómez-Román ${ }^{\mathrm{e}}$, Dolores Isla ${ }^{\mathrm{f}}$, José Ramírez ${ }^{\mathrm{g}}$, Luis Paz-Ares ${ }^{\mathrm{h}}$, \\ Julián Sanz ${ }^{i}$ y Enriqueta Felip ${ }^{j}$
}

\footnotetext{
a Servicio de Anatomía Patológica-Laboratorio de Dianas Terapéuticas, Hospital Universitario Sanchinarro, Madrid, España

b Servicio de Oncología Médica, Hospital Universitario La Paz, Madrid, España

c Servicio de Anatomía Patológica, Complejo Hospitalario Universitario A Coruña, A Coruña, España

¿ Servicio de Oncología Médica, Hospital Universitario Ramón y Cajal, Madrid, España

e Servicio de Anatomía Patológica, Hospital Universitario Marqués de Valdecilla, Universidad de Cantabria, IDIVAL, Santander, España

f Servicio de Oncología Médica, Hospital Clínico Lozano Blesa, Zaragoza, España

g Servicio de Anatomía Patológica, Hospital Clínic, IDIBAPS, CIBERES, Universidad de Barcelona, Barcelona, España

h Servicio de Oncología Médica, Instituto de Biomedicina de Sevilla-IBIS, Hospital Universitario Virgen del Rocío, Universidad de Sevilla, CSIC, Sevilla, España

i Servicio de Anatomía Patológica, Facultad de Medicina, Universidad Complutense de Madrid, Instituto de Investigación Sanitaria del Hospital Clínico San Carlos (IdISSC), Madrid, España

j Servicio de Oncología Médica, Hospital Universitari Vall d’Hebron, Barcelona, España
}

Recibido el 1 de octubre de 2014; aceptado el 24 de octubre de 2014

Disponible en Internet el 11 de diciembre de 2014

\section{PALABRAS CLAVE Receptor tirosina quinasa del linfoma anaplásico; Biomarcadores; Cáncer de pulmón;}

\begin{abstract}
Resumen En el año 2011 se inició un proyecto conjunto entre la Sociedad Española de Oncología Médica (SEOM) y la Sociedad Española de Anatomía Patológica (SEAP) para establecer unas recomendaciones basadas en la evidencia actual con respecto a la determinación de biomarcadores en pacientes con carcinoma de pulmón de célula no pequeña (CPCNP) avanzado. La mayoría de estas recomendaciones siguen siendo válidas; sin embargo, existen nuevas evidencias que hacen necesaria la actualización de algunos aspectos. En concreto, se modifica
\end{abstract}

\footnotetext{
* Autor para correspondencia.

Correo electrónico: flopezrios@hmhospitales.com (F. López-Ríos).
} 
Receptor del factor de crecimiento epidérmico

\section{KEYWORDS}

Anaplastic lymphoma receptor tyrosine

kinase;

Biomarkers;

Lung cancer;

Epidermal growth

factor receptor la recomendación de qué biomarcadores hay que analizar y en qué pacientes, y se define el manejo óptimo de la muestra tumoral así como las características del material mínimo necesario para la determinación de biomarcadores. Además, se revisan las técnicas adecuadas para la determinación de las mutaciones de EGFR y el reordenamiento de $A L K$, y se consensúa en qué situaciones se debe llevar a cabo una re-biopsia.

(c) 2014 SEAP y SEC. Publicado por Elsevier España, S.L.U. Todos los derechos reservados.

\section{Updated guidelines for biomarker testing in advanced non-small-cell lung cancer A national consensus of the Spanish Society of Pathology and the Spanish Society of Medical Oncology}

\begin{abstract}
In 2011 the Spanish Society of Medical Oncology (SEOM) and the Spanish Society of Pathology (SEAP) started a joint project to establish guidelines on biomarker testing in patients with advanced non-small-cell lung cancer (NSCLC) based on the current evidence. Most of these guidelines are still valid, but new evidence requires some aspects to be updated. Specifically, the recommendation about which biomarkers to test in which patients is being amended and the best way to manage tumour samples and minimum requirements for biomarker test material are defined. Suitable techniques for testing for EGFR mutations and ALK rearrangement are also reviewed, and a consensus is reached on which situations warrant re-biopsy.
\end{abstract}

(c) 2014 SEAP y SEC. Published by Elsevier España, S.L.U. All rights reserved.

\section{Introducción}

El cáncer de pulmón constituye la primera causa de muerte por cáncer, por lo que representa un importante problema sanitario. El hábito tabáquico es el principal responsable de cáncer de pulmón, y solo el $10-15 \%$ de estos tumores se diagnostican en pacientes no fumadores. Para decidir el tratamiento de los pacientes con carcinoma de pulmón no microcítico o de células no pequeñas (CPCNP) y enfermedad metastásica es necesario conocer el subtipo histológico y en la mayoría de los casos el resultado del análisis de biomarcadores. Es por ello que en el año 2011 se inició un proyecto conjunto entre la Sociedad Española de Oncología Médica (SEOM) y la Sociedad Española de Anatomía Patológica (SEAP) con el fin de definir unas recomendaciones conjuntas para el análisis de biomarcadores en pacientes con CPCNP y enfermedad avanzada a nivel nacional. En este primer consenso SEOM/SEAP participaron 5 patólogos y 5 oncólogos médicos con dedicación a patología torácica, que revisaron toda la bibliografía disponible y tras un consenso realizaron una serie de recomendaciones ${ }^{1}$. La mayoría de las recomendaciones del primer consenso SEOM/SEAP sobre biomarcadores en CPCNP siguen siendo válidas en la actualidad. Sin embargo, la publicación de nuevas evidencias, sobre todo en cuanto al valor predictivo de la determinación de la quinasa del linfoma anaplásico $(A L K)$, ha llevado a la elaboración de este segundo consenso SEOM/SEAP.

En este segundo consenso SEOM/SEAP han participado los mismos autores. La metodología ha consistido en una primera reunión presencial en la que se definieron las recomendaciones del primer consenso que precisaban una revisión y se formularon una serie de preguntas que se consideraron de interés. Posteriormente se revisó toda la literatura disponible y se contestaron todas las preguntas, incluyendo recomendaciones y referencias a cada una de ellas. Finalmente, en una segunda reunión presencial se debatió y aprobó este documento.

\section{¿Qué aspectos siguen siendo válidos del consenso anterior?}

En el primer consenso las recomendaciones se dividieron en aquellas relacionadas con aspectos clínicos, las relacionadas con aspectos anatomopatológicos y moleculares, y las que incluían aspectos comunes clínicos y relativos a los biomarcadores predictivos:

- Sobre las recomendaciones del primer consenso que analizaban aspectos clínicos sigue siendo válida la recomendación de determinar la mutación del receptor del factor de crecimiento epidérmico (EGFR) en los pacientes con CPCNP y enfermedad avanzada si presentan un carcinoma no escamoso, y en todos los pacientes no fumadores independientemente de la histología. Sin embargo, se consideró que la recomendación sobre la determinación de $A L K$ precisaba una actualización.

- La mayoría de las recomendaciones del primer consenso sobre aspectos anatomopatológicos siguen teniendo una vigencia total en la actualidad. Desde la publicación del primer consenso, la SEAP está implementando un programa de control de calidad sobre la determinación de $A L K$ y otro relativo a las nuevas tecnologías de secuenciación masiva.

- En cuanto a las recomendaciones sobre aspectos comunes clinicopatológicos, siguen siendo válidas las que se refieren a la optimización de la obtención de las muestras, la 


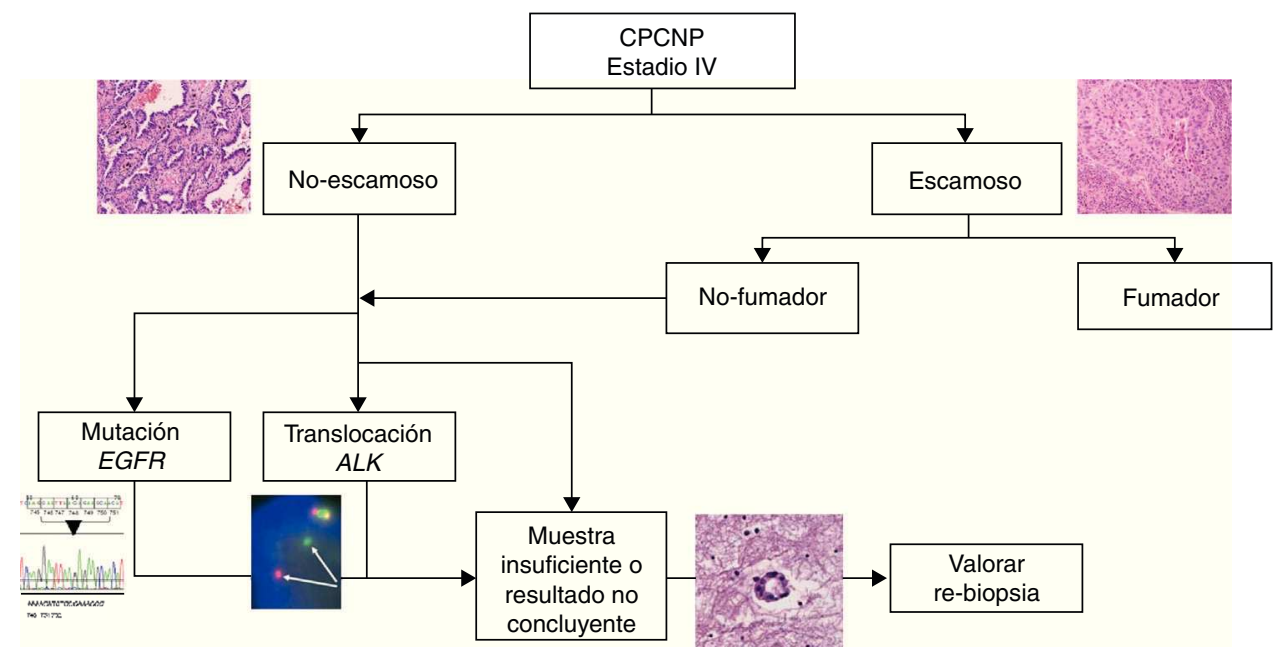

Figura 1 Algoritmo diagnóstico para los pacientes con CPCNP avanzado. ALK: quinasa del linfoma anaplásico; CPCNP: carcinoma de pulmón de célula no pequeña; EGFR: receptor del factor de crecimiento epidérmico.

preparación de las mismas, la información necesaria en los informes y los tiempos de respuesta aceptables (recomendación de tener los resultados en menos de 7 días laborables desde la recepción de la muestra).

Asimismo, en este segundo consenso SEOM/SEAP se actualiza la recomendación acerca de qué otros biomarcadores hay que analizar y en qué pacientes, se define el óptimo manejo de la muestra tumoral y las características del material mínimo necesario, se revisan las técnicas adecuadas para la determinación de las mutaciones de EGFR y los reordenamientos de $A L K$ y se consensúa en qué situaciones se debe realizar una re-biopsia. Finalmente, se analizan aspectos que pueden tener una gran relevancia en un futuro inmediato, como son las determinaciones moleculares en sangre y la utilización de técnicas de secuenciación masiva.

\section{¿Qué biomarcadores hay que analizar y en qué pacientes?}

En pacientes con CPCNP avanzado, la selección de tratamiento basado en los marcadores moleculares predictivos de eficacia ha modificado sustancialmente el enfoque clínico y las líneas de investigación de los últimos años.

\section{EGFR}

En nuestro medio, las mutaciones están presentes en el $10-16 \%$ de los pacientes con CPCNP avanzado ${ }^{2}$. Las más frecuentes (85-90\%) son las deleciones en el exón 19 y las mutaciones puntuales en el exón 21. En la actualidad disponemos de 3 fármacos (gefitinib, erlotinib y afatinib) que han demostrado claro beneficio en ensayos fase III aleatorizados en este contexto ${ }^{3-5}$. Por tanto, las principales guías clínicas recomiendan la prescripción de uno de ellos como tratamiento de primera línea en pacientes avanzados ${ }^{6,7}$. Durante un tiempo existió un cierto debate sobre el «perfil clínico» de los pacientes en los que se debería analizar la presencia de estas mutaciones. Hoy en día existe suficiente evidencia para recomendar esta determinación en pacientes con CPCNP no escamoso estadio Iv independientemente del hábito tabáquico, y en los no fumadores independientemente de la histología (fig. 1).

\section{Reordenamientos de $A L K$}

$A L K$ es un receptor tirosina quinasa que fue identificado por primera vez como parte de la translocación $\mathrm{t}(2 ; 5)$ asociada a la mayoría de los linfomas anaplásicos. Se calcula que el $2-7 \%$ de los pacientes con CPCNP tienen reordenamientos de $A L K^{8}$, principalmente translocaciones, siendo de nuevo más frecuente en pacientes con escasa o nula historia de tabaquismo. Estos pacientes tienden a ser jóvenes, y no hay diferencias de género. Se trata en su mayoría de adenocarcinomas, con frecuencia asociados a determinadas características morfológicas, por ejemplo células «en anillo de sello» o patrón cribiforme ${ }^{9}$. Los reordenamientos de ALK no suelen coexistir con las mutaciones de $E G F R^{10}$.

Existen diferentes inhibidores de $A L K$ en desarrollo clínico, si bien en Europa de momento solo está autorizado crizotinib. Este fármaco oral, con actividad frente a $A L K, c$ MET y ROS1, demostró beneficio significativo en términos de supervivencia libre de progresión (SLP) frente a quimioterapia, pemetrexed o docetaxel, en un estudio fase III llevado a cabo en 347 pacientes con cáncer de pulmón localmente avanzado o metastásico $A L K$-positivo previamente tratados con quimioterapia basada en platino ${ }^{11}$. Las medianas de SLP fueron 7,7 meses para los pacientes tratados con crizotinib y 3,0 meses para los pacientes tratados con quimioterapia (hazard ratio $[H R]: 0,49 ; p<0,001$ ). La tasa de respuesta objetiva (RO) también fue superior para los pacientes tratados con crizotinib (65\% vs. $20 \%$; $p<0,001)$. Los efectos adversos más frecuentes fueron alteraciones visuales, elevación de transaminasas, náuseas y vómitos.

Recientemente se han comunicado los resultados de un estudio fase III realizado en 343 pacientes con cáncer de pulmón no escamoso avanzado ALK-positivo y previamente 
no tratados, que fueron aleatorizados a recibir crizotinib $250 \mathrm{mg} 2$ veces al día o quimioterapia (pemetrexed con cisplatino o carboplatino $)^{12}$. Este estudio demostró la superioridad de crizotinib en comparación con quimioterapia en relación a la SLP (10,9 meses vs. 7,0 meses; HR: 0,454; $\mathrm{p}<0,0001)$. Asimismo, la tasa de RO también fue superior en los pacientes tratados con crizotinib ( $74 \%$ vs. $45 \%$; $\mathrm{p}<0,0001$ ).

Por último, también disponemos de los resultados de un estudio fase ı que evaluó la actividad de ceritinib, otro fármaco inhibidor de la tirosina quinasa (ITK) para el tratamiento de pacientes con CPCNP avanzado anaplásico $A L K$-positivo ${ }^{13}$. En este estudio ${ }^{14}, 255$ pacientes fueron tratados con $750 \mathrm{mg} /$ día de este fármaco. El efecto adverso más frecuente fue diarrea (84\%). En cuanto a los efectos adversos grado 3-4, los más frecuentes incluyeron elevación de las enzimas hepáticas alanina aminotransferasa (ALT) $(21 \%)$ y aspartato aminotransferasa (AST) (8\%). En este mismo ensayo clínico se evaluó la actividad de ceritinib en 121 pacientes previamente tratados con crizotinib. En ellos, se obtiene una SLP de 6,9 meses y una tasa de RO del 55,4\%. Esta tasa de RO se mantuvo con independencia del número de líneas previas de tratamientos recibidas (1-3). Asimismo, en este estudio también se analiza la evolución de 59 pacientes $A L K$-positivo no tratados previamente. En ellos no se alcanzó la mediana de SLP, y la tasa de RO fue del 69,5\%.

El estudio de reordenamientos de $A L K$ estaría indicado en pacientes con CPCNP no escamoso estadio Iv, independientemente del hábito tabáquico, y en los no fumadores independientemente del tipo morfológico (fig. 1). Es decir, tendría las mismas indicaciones que el estudio de las mutaciones de EGFR.

\section{¿Cuál es el tipo de muestra óptima y cómo se debe gestionar? ¿Se debe hacer un análisis simultáneo o secuencial?}

La muestra tumoral suele ser un material muy escaso sobre el que hay que definir 2 objetivos. El primero incluye obtener un diagnóstico anatomopatológico certero, y el segundo, la preservación de suficiente material para el posterior estudio de biomarcadores. El tejido obtenido por cirugía, broncoscopia o biopsia por punción es igual de válido, ya que el hecho de mayor relevancia es la cantidad de células tumorales presentes en la muestra. La citología se considera útil si se obtiene un bloque celular suficiente, ya que los estudios sobre extensiones solo resultan efectivos en centros de alta especialización. Es fundamental revisar todas las muestras tumorales de un paciente a la hora de decidir sobre cuál se van a realizar (o repetir) las determinaciones ${ }^{7}$.

El patólogo ha de utilizar la mínima cantidad de tejido para la tipificación del tumor y, como recomendación general (que puede ser modificada en función de las necesidades de cada caso), limitarse al uso de un máximo de 2 marcadores inmunohistoquímicos, en los casos sin clara diferenciación morfológica. El primer marcador necesario en el momento actual es el TTF-1, que tiene un rango de sensibilidad que oscila entre el $75-85 \%$ y una especificidad del $90-95 \%$ para los adenocarcinomas, siendo la positividad para carcinomas escamosos inapreciable $(0 \%)^{15}$. El segundo marcador aconsejado es $\mathrm{p} 40$, dada su mayor especificidad frente a p63, que sigue resultando útil. No obstante, hay que insistir que p63 marca hasta un $18 \%$ de los adenocarcinomas ${ }^{15}$. No se recomienda la realización de técnicas histoquímicas adicionales. Una vez realizado el diagnóstico de CPCNP no escamoso en estadio avanzado, se debe realizar el estudio de biomarcadores.

Es por todo ello que el material parafinado, sea de biopsia o bloque celular, ha de seguir un procedimiento (protocolo) de aprovechamiento tisular que permita no solo el diagnóstico anatomopatológico, sino también la realización de múltiples biomarcadores predictivos. Para evitar la pérdida de tejido cada vez que se coloca el bloque de tejido en el micrótomo conviene recordar 2 premisas: a) cuantas menos veces se ponga el bloque de parafina en el micrótomo, más se aprovecha el tejido, y b) el orden en que se jerarquicen los biomarcadores es importante, ya que el tejido se va agotando $^{16,17}$. La secuencia de pasos que proponemos realizar es la siguiente: una sección inicial teñida (hematoxilinaeosina [H\&E]) para obtener el primer diagnóstico, que permita programar (solo si la diferenciación glandular o escamosa no es evidente) 2 cortes para la realización de las pruebas inmunohistoquímicas, una serie de cortes para la extracción de ácido desoxirribonucleico (ADN) (el número y el grosor de los mismos dependerá del protocolo de extracción de ADN del laboratorio, aunque conviene resaltar que ya existe la posibilidad real de hacer los estudios mutacionales a partir de una sola sección de 5 micras), y por último 1-2 cortes para realizar las técnicas de determinación de ALK (fig. 2).

Un tema en discusión es si la determinación de ambos biomarcadores ha de realizarse simultáneamente o de forma secuencial. Aunque lo ideal sería hacerlos de forma simultánea, esto no es posible en todos los casos. Por tanto, una forma pragmática de ahorrar tejido y tiempo es planificar la realización simultánea, aunque las determinaciones se realicen de forma secuencial. Esto quiere decir que las secciones para el estudio tanto de EGFR como de $A L K$ se realizan al mismo tiempo, aunque el estudio de uno de los biomarcadores lo activemos solo en el caso de que el otro haya sido negativo.

\section{¿Qué mutaciones de EGFR se deben determinar y con qué técnicas?}

\section{Mutaciones de EGFR a determinar}

En general, existe acuerdo con la opinión del consenso del College of American Pathologists, International Association for the Study of Lung Cancer y Association for Molecular Pathology en cuanto a que las pruebas de mutaciones clínicas de EGFR deben ser capaces de detectar todas las mutaciones individuales que han sido informadas con una frecuencia de al menos un $1 \%$ de los adenocarcinomas de pulmón mutados en $E G F R^{18,19}$. La realización de pruebas para detectar solamente las 2 mutaciones principales ya no se considera aceptable (tabla 1). Se deben analizar las mutaciones de los exones 18-21 del dominio tirosina quinasa de EGFR. Dos tipos de mutaciones componen el $90 \%$ de todas las mutaciones activadoras de EGFR, las deleciones en el exón 19 (rodeando los codones 746-750) y la mutación en el exón 21 (L858R). Las mutaciones puntuales en los exones $18 \mathrm{y}$ 


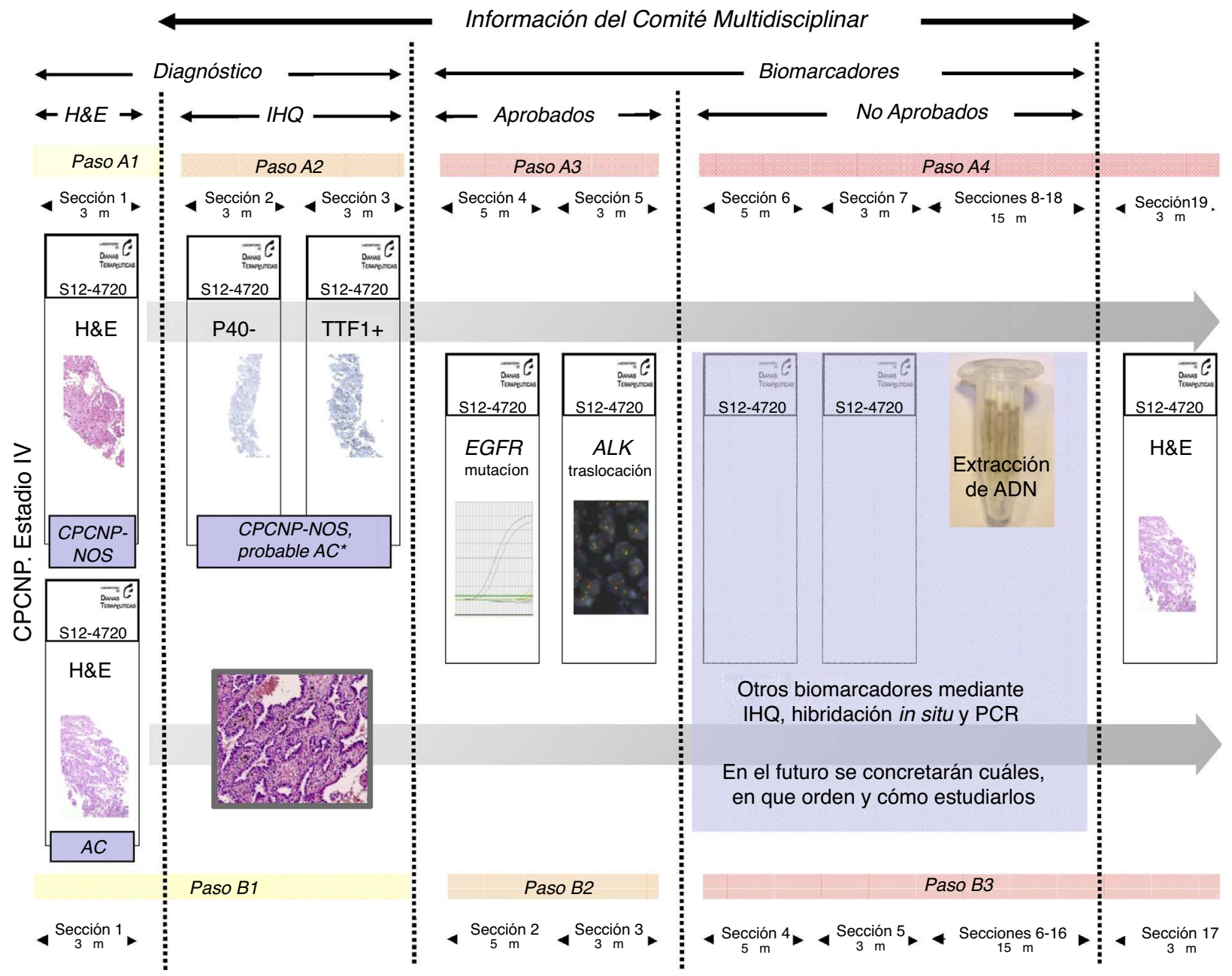

Figura 2 Protocolo para el estudio de múltiples biomarcadores en muestras de pacientes con CPCNP avanzado.

La ruta superior (A) es para casos que requieran IHQ clasificatoria. La ruta inferior (B) se aplicaría en muestras en donde no es necesario este paso, al objetivarse claramente diferenciación glandular con la H\&E.

AC: adenocarcinoma; ADN: ácido desoxirribonucleico; ALK: quinasa del linfoma anaplásico; CPCNP: carcinoma de pulmón de célula no pequeña; EGFR: receptor del factor de crecimiento epidérmico; H\&E: hematoxilina-eosina; IHQ: inmunohistoquímica; NOS: (de tipo) no especificado; PCR: reacción en cadena de la polimerasa.

Modificado de Conde et al. ${ }^{16}$.

20 (principalmente T790M) y las inserciones en el exón 20 suman otro $2-5 \%$ y $5-10 \%$, respectivamente.

Aunque existe una tendencia creciente hacia la extensiva caracterización molecular de los tumores, se recomienda informar de las mutaciones poco habituales de forma separada, con un comentario acerca de su poco clara o incierta significación clínica. Algunas publicaciones recientes pueden ayudar a entender el significado clínico de algunas de estas mutaciones raras ${ }^{20,21}$.

\section{Técnicas para determinar las mutaciones en EGFR}

Se pueden distinguir métodos de detección sistemática, que detectan todas las mutaciones en los exones 18-21, incluyendo las variantes nuevas y los métodos «diana», que detectan mutaciones específicas. Aunque este es un entorno muy cambiante, se pueden hacer algunas recomendaciones prácticas. Si es posible, lo ideal es utilizar un método de mayor sensibilidad que la secuenciación directa, como la reacción en cadena de la polimerasa (PCR) en tiempo real (las recientes recomendaciones del National Institute for Health and Care Excellence [NICE] seleccionan 2 métodos concretos). La secuenciación directa solo debería realizarse si tenemos al menos un $50 \%$ de células tumorales. En cuanto a los informes de laboratorio, deben especificar siempre qué mutaciones han sido detectadas sistemáticamente y qué sensibilidad tienen los métodos de detección utilizados.

\section{¿Con qué técnicas se debe determinar ALK?}

En la actualidad existen fundamentalmente 3 técnicas que permiten detectar el reordenamiento del gen $A L K$ en las muestras clínicas. Se trata de la inmunohistoquímica, de la hibridación in situ fluorescente (FISH) y la PCR con retrotranscripción (RT-PCR). La única prueba aprobada por la Food and Drug Administration (FDA) estadounidense es FISH 
Tabla 1 Mutaciones de EGFR

\begin{tabular}{|c|c|c|c|}
\hline Exón & Codón & Mutaciones & $\begin{array}{l}\% \text { de todas las } \\
\text { mutaciones } \\
\text { en EGFR }\end{array}$ \\
\hline \multirow[t]{2}{*}{18} & 709 & $\begin{array}{l}\text { E709K, E709A, } \\
\text { E709G, E709V, } \\
\text { E709D, E709Q }\end{array}$ & 1 \\
\hline & 719 & $\begin{array}{l}\text { G719S, } \\
\text { G719C,G719A, } \\
\text { G719D }\end{array}$ & $2-5$ \\
\hline \multirow[t]{2}{*}{19} & $739-744$ & Inserciones & 1 \\
\hline & $746-753$ & $\begin{array}{l}\text { Deleciones: } 9 \text {, } \\
\text { 12, } 15,18 \text { or } 24 \\
\text { bp }\end{array}$ & 45 \\
\hline \multirow[t]{3}{*}{20} & $\begin{array}{l}763,764 \\
767-774\end{array}$ & $\begin{array}{l}\text { Inserciones } \\
\text { Inserciones }{ }^{a} \text { : } \\
3,6,9,12 \text { bp }\end{array}$ & $5-10$ \\
\hline & 768 & S768I & $1-2$ \\
\hline & 790 & $\mathrm{~T} 790 \mathrm{M}^{\mathrm{a}}$ & 2 \\
\hline \multirow[t]{3}{*}{21} & 858 & L858R & 40 \\
\hline & & L858M & Rara \\
\hline & 861 & L861Q, L861R & $2-5$ \\
\hline
\end{tabular}

EGFR: receptor del factor de crecimiento epidérmico.

a Mutaciones asociadas con resistencia a inhibidores de la tirosina quinasa (ITK).

con la sonda comercial Vysis ${ }^{\circledR}$ ALK Break-Apart FISH Probe Kit (Abbott Molecular, Inc.), que recibió la aprobación como prueba acompañante del fármaco crizotinib ${ }^{22}$. En este sentido es interesante resaltar que un escáner de FISH ha recibido recientemente aprobación por la FDA para la interpretación de la sonda de $A L K$ mencionada anteriormente ${ }^{23}$. Por otro lado, existen alteraciones en el número de copias del gen $A L K$ que no parecen relacionadas con la buena respuesta al fármaco específico, si bien es preciso estudiar en profundidad los casos con un incremento en el número de copias para no perder posibles casos de presentación atípica $^{24,25}$.

La aparición de clones con una sensibilidad apropiada para su uso mediante inmunohistoquímica, como los D5F3 y 5A4, y el uso de técnicas de amplificación para intensificar la señal inmunohistoquímica a base de polímeros, ha provocado que se proponga su uso como método de cribado previo a la confirmación con el método FISH ${ }^{19,26}$. La mayor parte de las series publicadas muestran una concordancia muy elevada entre las técnicas FISH e inmunohistoquímica $22,27-29$, si bien existen falsos positivos y negativos con ambas metodologías. Probablemente el riesgo mayor es que la sensibilidad disminuya, por lo que es muy importante: a) intentar controlar los parámetros pre-analíticos, que afectan sobre todo a la inmunohistoquímica, y b) ser conscientes de la dificultad interpretativa de FISH (es sensato verificar los resultados en casos de patrones inusuales). Como regla práctica, recomendamos realizar 2 métodos de estudio del gen $A L K$ cuando existan incertidumbres de cualquier naturaleza sobre el resultado de la determinación. En nuestra experiencia las más habituales han sido: una dudosa respuesta al tratamiento de un caso informado como positivo, las determinaciones en los carcinomas escamosos, una tinción inmunohistoquímica no claramente granular y las polisomías y monosomías en el estudio de FISH. En cuanto a la técnica molecular de RT-PCR, si bien puede mostrar una sensibilidad y una especificidad adecuadas, no está recomendada por el colegio estadounidense de patólogos ${ }^{19}$.

\section{¿En qué situaciones se debe re-biopsiar al paciente?}

La realización de una nueva toma de material del tumor se contempla habitualmente en 2 situaciones: a) cuando se está realizando el diagnóstico inicial del mismo, y b) en ciertas circunstancias cuando se produce una progresión de la enfermedad. En ambos casos, su objetivo es la orientación hacia la mejor opción de tratamiento.

\section{Realización de una re-biopsia al inicio del diagnóstico}

La decisión de realizar una re-biopsia debe fundamentarse en 3 pilares que darán una estimación del beneficio real: a) las características clínicas del paciente; b) los aspectos anatomopatológicos del tumor, y c) los riesgos de la rebiopsia. Desde el punto de vista de la indicación, se debe valorar si la re-biopsia va a ser imprescindible para tomar la mejor decisión terapéutica. Con respecto a los riesgos de este procedimiento, habrá que estimar la dificultad técnica de su realización con su potencial morbilidad y la demora en el inicio del tratamiento que puede implicar. Por todo ello, será fundamental informar adecuadamente al paciente de los pros y contras de la nueva toma de material tumoral, y las razones y objetivos que llevan a esa decisión. Con todas estas consideraciones, se recomienda hacer una re-biopsia del tumor cuando existen algunas de las siguientes circunstancias: a) siempre que el resultado del análisis molecular no haya sido informativo o no se disponga de material tumoral suficiente para su realización, y las características clínicopatológicas del paciente y del tumor orienten a la posible presencia de una diana terapéutica; ejemplos de ello pueden ser pacientes que nunca hayan sido fumadores o con edad inferior a la presentación habitual de la enfermedad, o características patológicas sugestivas, como la presencia de células en anillo de sello ${ }^{30}$ o un patrón micropapilar ${ }^{31}$, y b) cuando exista discrepancia entre el perfil clínico del paciente y el resultado anatomopatológico obtenido. Así, cuando el diagnóstico sea de un carcinoma de tipo escamoso o de células pequeñas en un paciente que nunca haya sido fumador o de edad muy joven, habría que revisar el estudio anatomopatológico o valorar, en cualquier caso, la realización de los estudios moleculares establecidos para los tumores de histología no escamosa. De igual modo, cuando el diagnóstico inicial sea compatible con un carcinoma no microcítico de tipo no especificado (NOS) puede hacerse este mismo planteamiento.

\section{Realización de re-biopsia ante la progresión de la enfermedad}

En caso de progresión de la enfermedad, puede realizarse una re-biopsia en 2 situaciones: a) cuando ha habido una evolución anormal de la enfermedad y se quiere confirmar 
el diagnóstico inicial, y b) cuando se quiere realizar un nuevo estudio molecular que oriente el tratamiento a seguir ${ }^{32}$. Se recomienda solicitar la realización de estudios moleculares en las re-biopsias para encontrar mutaciones tras la resistencia adquirida a ITK-EGFRs ${ }^{33}$. La mutación T790M en el gen EGFR es la más común (50-70\%) y existen fármacos con eficacia probada frente a esta mutación como como AZD9291 y CO-1686 34,35 . En los casos en que existe un reordenamiento de $A L K$ y que progresan a un inhibidor, la biopsia podría determinar la causa de la progresión, aunque todavía no se ha definido el tratamiento a seguir en esta situación ${ }^{36}$.

\section{¿Qué otros biomarcadores tienen interés hoy en día?}

En CPCNP están siendo investigados otros biomarcadores por su potencial interés como predictores de la eficacia a determinados fármacos en el contexto de ensayos clínicos, pero de momento no hay evidencia científica para su recomendación en la práctica clínica ${ }^{19,37,38}$.

\section{Mutación de KRAS}

KRAS es un gen que aparece mutado en alrededor del $20 \%$ de todos los casos de CPCNP, especialmente en adenocarcinomas y en pacientes fumadores. Es la mutación oncogénica más frecuente y su valor pronóstico no ha sido demostrado claramente. En la actualidad se encuentran en investigación diferentes fármacos, destacando especialmente los que actúan en niveles inferiores de la vía de señalización RAS, como los inhibidores de MEK $1 / 2$, cuya eficacia también se está evaluando, y los inhibidores de la vía de señalización $\mathrm{PI3K} / \mathrm{AKT} / \mathrm{mTOR}^{39}$.

\section{Alteración de MET}

MET es un receptor tirosina quinasa activado por su ligando específico natural, el factor de crecimiento hepatocitario (HGFR) situado en el cromosoma 7q21-q31. El gen MET puede estar mutado raramente en CPCNP (1\%), o amplificado (3-7\%). También se ha descrito la sobreexpresión del receptor (25-75\%), condicionando un peor pronóstico. Estas alteraciones aparecen en cualquier histología de CPCNP y son independientes de la presencia de mutaciones de KRAS o EGFR. Entre el $10-20 \%$ de los pacientes con tumores EGFR mutados adquieren resistencia a los ITK-EGFRs a través de la amplificación de MET. Se encuentran en investigación algunos inhibidores de MET, anticuerpos monoclonales o ITK, administrados en combinación con otras terapias dirigidas o quimioterapia, o en monoterapia.

\section{Translocación de ROS1}

ROS1 es un gen que codifica un receptor tirosina quinasa que aparece translocado en el 1-2\% de los pacientes con CPCNP, sobre todo en no fumadores, en pacientes con adenocarcinoma, jóvenes y sin mutaciones en los genes EGFR, $K R A S, B R A F, H E R 2$ o reordenamiento de $A L K^{40}$. Crizotinib ha demostrado actividad en estos pacientes ${ }^{41}$.

\section{Mutación de BRAF}

$B R A F$ es un gen que codifica una proteína con actividad serina-treonina quinasa, que se encuentra en la vía de señalización de MAPK a nivel inferior de RAS. Su mutación está presente en el 1-3\% de los CPCNP, en más de la mitad de los casos es una mutación V600E, y aparece sobre todo en adenocarcinomas y en pacientes fumadores y ex fumadores ${ }^{42}$. Están siendo investigados diferentes inhibidores de BRAF que han presentado actividad en estudios iniciales, considerándose asimismo la estrategia de su asociación a inhibidores de MEK, especialmente para pacientes con mutación no V600E.

\section{Translocación de RET}

El gen RET codifica el receptor tirosina quinasa, y su reordenamiento aparece con una incidencia de $1,4-2 \%$ en los CPCNP. Se detecta fundamentalmente en adenocarcinomas, pacientes no fumadores y jóvenes y en ausencia de otras alteraciones genéticas (EGFR, KRAS, BRAF, HER2 o translocación $A L K)^{43}$. En la actualidad están siendo investigados diferentes inhibidores de $R E T$, la mayoría de los cuales son agentes frente a múltiples quinasas.

\section{Mutación de HER2}

HER2 es una proteína tirosina quinasa de membrana de la familia ERBB, se sobreexpresa en el $20 \%$ de los CPCNP, pero solo se han descrito mutaciones o amplificación del gen en un 2-4\% de los casos. La mutación se observa especialmente en las mujeres, en los pacientes no fumadores, en los adenocarcinomas y en los pacientes de raza asiática. Generalmente se trata de la inserción en el exón 20 y es mutuamente excluyente con las mutaciones de EGFR o KRAS ${ }^{44}$. En estudios iniciales, especialmente los agentes ITK irreversibles de HER2 y EGFR han demostrado actividad en pacientes con CPCNP, y se está evaluando su asociación con otras terapias dirigidas como los inhibidores de mTOR, debido a la asociación entre la mutación de HER2 y la activación preferencial de la vía $A K T / m T O R$.

\section{Otros potenciales biomarcadores}

Otros potenciales biomarcadores de interés, particularmente en carcinomas escamosos, son las mutaciones o la amplificación de PI3KCA, la amplificación de FGFR1, la expresión de PD-L1 o las mutaciones de DDR2.

\section{¿Qué otras técnicas tienen interés hoy en día?}

Ante el reto que se nos presenta sobre la necesidad de determinar múltiples biomarcadores para optimizar la eficacia de las nuevas terapias, los avances técnicos recientes con sistemas de nueva generación que permiten la secuenciación masiva de genes en una sola prueba suponen una buena oportunidad para abordar este nuevo escenario. Hay que recalcar que estos son estudios de docenas de genes y miles de mutaciones (coloquialmente referida como «secuenciación masiva dirigida»), pero que no estamos hablando de 
secuenciación exómica o genómica completa. Estas tecnologías son muy robustas para el estudio de mutaciones, pero pueden presentar problemas de sensibilidad y especificidad para estudiar inserciones o pequeñas deleciones. En general es muy recomendable, o incluso imprescindible en muchas determinaciones, disponer de tejido normal (o sangre) acompañante. Aunque técnicamente es factible el estudio de translocaciones, no está claro su rendimiento y robustez en muestras que han sido fijadas en formol e incluidas en parafina. Una buena opción sería realizar las determinaciones más prevalentes y/o realmente imprescindibles por los métodos convencionales y reservar estos abordajes más masivos para los casos pan-negativos, como por ejemplo EGFR, ALK, KRAS, RET, ROS1, BRAF y HER2 negativos. Es interesante resaltar que las amplificaciones tratables (estos es, MET o HER2) ocurren típicamente en estos adenocarcinomas de pulmón «oncogén-negativos» ${ }^{45}$. Por tanto, es imprescindible asegurarnos en este último subgrupo de que las amplificaciones están adecuadamente estudiadas mediante secuenciación masiva o FISH.

\section{¿Cuál es el material mínimo necesario para la determinación de biomarcadores?}

La cantidad mínima de células neoplásicas que debe contener una muestra para el diagnóstico de cáncer de pulmón y la realización de estudios moleculares es variable, pues depende de muchos factores, como son: a) el tipo de método diagnóstico a utilizar; b) la presencia de elementos estromales-inflamatorios abundantes; c) la existencia de necrosis, hemorragia o fibrosis extensa; d) la calidad del tejido, la preservación antigénica o la integridad del ADN/ácido ribonucleico (ARN), y e) la existencia de alteraciones genómicas como aneuploidías, polisomías y amplificaciones. Según los casos, es relevante disponer de un número absoluto de células tumorales representativas, o bien que la proporción de las mismas alcance un valor determinado. Para estudios citogenéticos se recomienda un mínimo de 100 células tumorales, por cuestiones técnicas, en tanto que para estudios moleculares es necesario disponer de al menos un 30\% de células tumorales (para la determinación mediante secuenciación directa) o un $5 \%$ de células tumorales (para la determinación mediante PCR en tiempo real). Existe tecnología que permite detectar mutaciones cuando la población tumoral supone un $10 \%$ o incluso menos aún $(1-0,1 \%)$. Aunque hay que tener cuidado con estos métodos untrasensibles, pues se pueden dar artefactos y aportar falsos positivos con mayor frecuencia ${ }^{19}$. Se asume que, con las tecnologías disponibles, son suficientes cantidades de 250-500 ng de ADN/ARN para realizar los estudios moleculares que exploren diferentes genes simultáneamente ${ }^{46}$.

Es crucial que las instituciones establezcan las estrategias necesarias para optimizar los estudios de biomarcadores ${ }^{47}$, y que cada laboratorio determine su propio umbral de sensibilidad analítica, o límite de detección, para validar sus procedimientos. Para los casos de bajo rendimiento se debe valorar la posibilidad de realizar una nueva biopsia ${ }^{19}$. Si el estudio resultante es negativo y el porcentaje tumoral está cerca del límite de detección de nuestra metodología, es más sensato que el diagnóstico sea «no concluyente», para dar la oportunidad de repetir la determinación si tiene lugar una re-biopsia, como ya se ha descrito en apartados anteriores.

\section{¿Cuáles son las aplicaciones de la biopsia líquida?}

El perfil molecular de interés de los tumores sólidos se obtiene en la actualidad, como se ha comentado previamente, a partir de especímenes quirúrgicos o de biopsias. Sin embargo, este último procedimiento no siempre puede llevarse a cabo de forma rutinaria debido a su carácter invasivo. Adicionalmente, la información obtenida a partir de una sola biopsia proporciona una instantánea espacial y temporalmente limitada de un tumor y podría no reflejar su heterogeneidad. Como alternativa a la muestra tisular, hay un creciente interés en el estudio de biopsias líquidas o muestras sanguíneas del paciente, mediante la caracterización molecular de células tumorales circulantes (CTC) y el escrutinio del ADN libre circulante (cfDNA) en el suero ${ }^{48,49}$.

El estudio de las CTC en cáncer de pulmón ha sido menos exhaustivo que en otros tipos tumorales ${ }^{50}$. Su recuento pudiera tener utilidad pronóstica y en la monitorización de la respuesta a tratamientos. En los últimos años han visto la luz algunos trabajos mostrando la posibilidad de caracterización genética y citogenética de CTC capturadas con tecnologías sofisticadas como los filtros microfluídicos («CTC-Chip»). En un estudio realizado en el Massachusetts General Hospital se mostró una sensibilidad del 95\% en la detección de mutaciones activadoras de EGFR utilizando Scorpion Amplification Refractory Mutation System (SARMS) en 20 pacientes, de los cuales 11 presentaban asimismo la mutación de resistencia $\mathrm{T} 790 \mathrm{M}^{51}$.

La utilidad potencial del análisis del cfDNA sérico incluye la detección precoz, la monitorización de la respuesta al tratamiento y la detección de recidiva, así como particularmente la determinación de anomalías moleculares condicionantes del tratamiento dirigido a dianas moleculares y el análisis de la evolución genotípica del tumor tras la presión terapéutica ${ }^{4,49}$. En este último sentido, la sensibilidad depende del estadio y de la carga tumoral, así como de factores técnicos. La sensibilidad para la determinación de alteraciones concretas como las mutaciones de EGFR o KRAS en CPCNP con tecnologías avanzadas (PCR digital, beads, emulsions, amplification and magnetics [BEAMing] y nextgeneration sequencing [NGS]) es inferior al $0,01 \%$, de modo que pudieran identificarse alteraciones genéticas de interés en $\geq 90 \%$ de los pacientes con enfermedad en estadio Iv ${ }^{4,52}$.

En el momento actual, la caracterización de biopsias líquidas ha de considerarse esencialmente en el contexto de la investigación. Pudiera contemplarse excepcionalmente la utilidad de estos procedimientos, particularmente la identificación de mutaciones de EGFR en cfDNA, en el caso de tipificación molecular incompleta de un CPCNP avanzado y dificultad técnica o contraindicación médica para el muestreo convencional.

\section{Conclusiones}

Es ya una realidad que en los pacientes con CPCNP avanzado es necesaria no solo la información anatomopatológica 
sino también el resultado de biomarcadores predictivos. Hoy en día existe suficiente evidencia para recomendar el estudio de las mutaciones de EGFR y de la translocación de ALK en pacientes con CPCNP no escamoso estadio Iv independientemente del hábito tabáquico, y en los no fumadores independientemente de la histología. Aunque lo ideal sería hacer los 2 biomarcadores de forma simultánea, esto no es posible en todos los casos. Por tanto, una forma pragmática de ahorrar tejido y tiempo es planificar la realización simultánea aunque las determinaciones se realicen de forma secuencial. Los avances tecnológicos ya permiten trabajar con protocolos de priorización de la muestra para conseguir estos objetivos, siempre encuadrados dentro de controles de calidad y cursos de formación. Realizar todo el proceso de análisis morfológico y patológico de la muestra y de los marcadores predictivos con rapidez y calidad solo es factible con un trabajo multidisciplinar eficaz.

\section{Responsabilidades éticas}

Protección de personas y animales. Los autores declaran que para esta investigación no se han realizado experimentos en seres humanos ni en animales.

Confidencialidad de los datos. Los autores declaran que en este artículo no aparecen datos de pacientes.

Derecho a la privacidad y consentimiento informado. Los autores declaran que en este artículo no aparecen datos de pacientes.

\section{Financiación}

La SEOM y la SEAP agradecen el apoyo financiero de este proyecto mediante becas sin restricción de AstraZeneca, Boehringer Ingelheim, Lilly, Novartis, Pfizer y Roche.

\section{Conflicto de intereses}

Los autores declaran que, en el momento de la redacción y revisión del texto, desconocían el nombre de los laboratorios que han apoyado económicamente este proyecto, por lo que este apoyo no ha influido en el contenido de este artículo.

\section{Agradecimientos}

Los autores agradecen la asistencia editorial de Ana Martín, de HealthCo (Madrid, España), en el desarrollo de este manuscrito. F. López-Ríos agradece a B. Angulo y E. Conde su contribución a las terapias dirigidas en el carcinoma de pulmón.

\section{Bibliografía}

1. Garrido P, de Castro J, Concha A, Felip E, Isla D, Lopez-Rios F, et al. Guidelines for biomarker testing in advanced non-smallcell lung cancer. A national consensus of the Spanish Society of Medical Oncology (SEOM) and the Spanish Society of Pathology (SEAP). Clin Transl Oncol. 2012;145:338-49.
2. Rosell R, Moran T, Queralt C, Porta R, Cardenal F, Camps C, et al. Screening for epidermal growth factor receptor mutations in lung cancer. N Engl J Med. 2009;361:958-67.

3. Mok TS, Wu YL, Thongprasert S, Yang CH, Chu DT, Saijo N, et al. Gefitinib or carboplatin-paclitaxel in pulmonary adenocarcinoma. N Engl J Med. 2009;361:947-57.

4. Rosell R, Carcereny E, Gervais R, Vergnenegre A, Massuti B, Felip E, et al. Erlotinib versus standard chemotherapy as firstline treatment for European patients with advanced EGFR mutation-positive non-small-cell lung cancer (EURTAC): A multicentre, open-label, randomised phase 3 trial. Lancet Oncol. 2012;133:239-46.

5. Sequist LV, Yang JC, Yamamoto N, O’Byrne K, Hirsh V, Mok T, et al. Phase III study of afatinib or cisplatin plus pemetrexed in patients with metastatic lung adenocarcinoma with EGFR mutations. J Clin Oncol. 2013;31:3327-34.

6. Camps C, Felip E, Garcia-Campelo R, Trigo JM, Garrido P. SEOM clinical guidelines for the treatment of non-small cell lung cancer (NSCLC) 2013. Clin Transl Oncol. 2013;15:977-84.

7. Kerr KM, Bubendorf L, Edelman MJ, Marchetti A, Mok T, Novello S, et al. Panel Members: Panel Members. Second ESMO consensus conference on lung cancer: pathology and molecular biomarkers for non-small-cell lung cancer. Ann Oncol. 2014;25:1681-90.

8. Shaw AT, Yeap BY, Mino-Kenudson M, Digumarthy SR, Costa DB, Heist RS, et al. Clinical features and outcome of patients with non-small-cell lung cancer who harbor EML4-ALK. J Clin Oncol. 2009;27:4247-53.

9. Fan L, Feng Y, Wan H, Shi G, Niu W. Clinicopathological and demographical characteristics of non-small cell lung cancer patients with ALK rearrangements: a systematic review and meta-analysis. PLoS One. 2014 [consultado Jul 2014]. Disponible en: http://www.plosone.org/ article/info\%3Adoi\%2F10.1371\%2Fjournal.pone.0100866

10. Kris MG, Johnson BE, Berry LD, Kwiatkowski DJ, lafrate AJ, Wistuba II, et al. Using multiplexed assays of oncogenic drivers in lung cancers to select targeted drugs. JAMA. 2014;311:1998-2006.

11. Shaw AT, Kim DW, Nakagawa K, Seto T, Crino L, Ahn MJ, et al. Crizotinib versus chemotherapy in advanced ALK-positive lung cancer. N Engl J Med. 2013;368:2385-94.

12. Mok T, Kim DW, Wu YL, Solomon BJ, Nakagawa K, Mekhail $\mathrm{T}$, et al. First-line crizotinib versus pemetrexed-cisplatin or pemetrexed-carboplatin in patients (pts) with advanced ALKpositive non-squamous non-small cell lung cancer (NSCLC): Results of a phase III study (PROFILE 1014). J Clin Oncol. 2014;32(5s) (suppl; abstr 8002).

13. Shaw AT, Kim DW, Mehra R, Tan DS, Felip E, Chow LQ, et al, Ceritinib in ALK-rearranged non-small-cell lung cancer. N Engl J Med. 2014;370:1189-97.

14. Kim D-W, Mehra R, Tan DS-W, Felip E, Chow LQM, Camidge $\mathrm{DR}$, et al. Ceritinib in advanced anaplastic lymphoma kinase (ALK)-rearranged (ALK+) non-small cell lung cancer (NSCLC): Results of the ASCEND-1 trial. ASCO Meeting Abstract 2014;3215_suppl:8003.

15. Nonaka D. A study of DeltaNp63 expression in lung non-small cell carcinomas. Am J Surg Pathol. 2012;36:895-9.

16. Conde E, Angulo B, Izquierdo E, Paz-Ares L, Belda-Iniesta $C$, Hidalgo $M$, et al. Lung adenocarcinoma in the era of targeted therapies: Histological classification, sample prioritization, and predictive biomarkers. Clin Transl Oncol. 2013;15: 503-8.

17. Lopez-Rios F, Conde E, Angulo B, Martinez R, McGready C, O'Hara G, et al. A prospective protocol for simultaneous genomic testing in patients with non-small cell lung cancer. J Thorac Oncol. 2013;8:S1074.

18. EGFR-TK mutation testing in adults with locally advanced or metastatic non-small-cell lung cancer. NICE diagnostics 
guidance 9 [consultado May 2014]. Disponible en: http:// guidance.nice.org.uk/nicemedia/live/14251/64893/64893.pdf

19. Lindeman NI, Cagle PT, Beasley MB, Chitale DA, Dacic S, Giaccone $\mathrm{G}$, et al. Molecular testing guideline for selection of lung cancer patients for EGFR and ALK tyrosine kinase inhibitors: Guideline from the College of American Pathologists, International Association for the Study of Lung Cancer, and Association for Molecular Pathology. Arch Pathol Lab Med. 2013;137:828-60.

20. Arcila ME, Nafa K, Chaft JE, Rekhtman N, Lau C, Reva BA, et al. EGFR exon 20 insertion mutations in lung adenocarcinomas: Prevalence, molecular heterogeneity, and clinicopathologic characteristics. Mol Cancer Ther. 2013;12:220-9.

21. He M, Capelletti M, Nafa K, Yun CH, Arcila ME, Miller VA, et al. EGFR exon 19 insertions: A new family of sensitizing EGFR mutations in lung adenocarcinoma. Clin Cancer Res. 2012;18:1790-7.

22. Hutarew G, Hauser-Kronberger C, Strasser F, Llenos IC, Dietze $\mathrm{O}$. Immunohistochemistry as a screening tool for ALK rearrangement in NSCLC: evaluation of five different ALK antibody clones and ALK FISH. Histopathology. 2014;65:398-407.

23. FDA clears BioView Duet ${ }^{T M}$ system for automated ALK FISH scanning of lung cancer tissue specimens [consultado Jun 2014] Disponible en: http://www.bioview.co.il/htmls/page_70. aspx?c0 $=12677 \& b s p=12624$

24. Camidge DR, Skokan M, Kiatsimkul P, Helfrich B, Lu X, Baron AE, et al. Native and rearranged ALK copy number and rearranged cell count in non-small cell lung cancer: implications for ALK inhibitor therapy. Cancer. 2013;119:3968-75.

25. Salido M, Pijuan L, Martinez-Aviles L, Galvan AB, Canadas I, Rovira A, et al. Increased ALK gene copy number and amplification are frequent in non-small cell lung cancer. J Thorac Oncol. 2011;61:21-7.

26. Thunnissen E, Bubendorf L, Dietel M, Elmberger G, Kerr K, Lopez-Rios F, et al. EML4-ALK testing in non-small cell carcinomas of the lung: A review with recommendations. Virchows Arch. 2012;46:245-57.

27. Selinger Cl, Rogers TM, Russell PA, O'Toole S, Yip P, Wright GM, et al. Testing for ALK rearrangement in lung adenocarcinoma: A multicenter comparison of immunohistochemistry and fluorescent in situ hybridization. Mod Pathol. 2013;26:1545-53.

28. Wynes MW, Sholl LM, Dietel M, Schuuring E, Tsao MS, Yatabe $Y$, et al. An international interpretation study using the ALK $\mathrm{IHC}$ antibody D5F3 and a sensitive detection kit demonstrates high concordance between ALK IHC and ALK FISH and between evaluators. J Thorac Oncol. 2014;95:631-8.

29. Zwaenepoel K, Van Dongen A, Lambin S, Weyn C, Pauwels P. Detection of ALK expression in non-small-cell lung cancer with ALK gene rearrangements - comparison of multiple immunohistochemical methods. Histopathology. 2014;65:539-48.

30. Nishino M, Klepeis VE, Yeap BY, Bergethon K, Morales-Oyarvide V, Dias-Santagata D, et al. Histologic and cytomorphologic features of ALK-rearranged lung adenocarcinomas. Mod Pathol. 2012;25:1462-72.

31. Motoi N, Szoke J, Riely GJ, Seshan VE, Kris MG, Rusch VW, et al. Lung adenocarcinoma: modification of the 2004 WHO mixed subtype to include the major histologic subtype suggests correlations between papillary and micropapillary adenocarcinoma subtypes, EGFR mutations and gene expression analysis. Am J Surg Pathol. 2008;32:810-27.

32. Arcila ME, Oxnard GR, Nafa K, Riely GJ, Solomon SB, Zakowski $M F$, et al. Rebiopsy of lung cancer patients with acquired resistance to EGFR inhibitors and enhanced detection of the T790M mutation using a locked nucleic acid-based assay. Clin Cancer Res. 2011;17:1169-80.

33. Hata A, Katakami N, Yoshioka H, Takeshita J, Tanaka K, Nanjo S, et al. Rebiopsy of non-small cell lung cancer patients with acquired resistance to epidermal growth factor receptor-tyrosine kinase inhibitor: Comparison between T790M mutation-positive and mutation-negative populations. Cancer. 2013;119:4325-32.
34. Janne PA, Ramalingam SS, Yang JC-H, Ahn M-J, Kim D-W, Kim $\mathrm{S}-\mathrm{W}$, et al. Clinical activity of the mutant-selective EGFR inhibitor AZD9291 in patients (pts) with EGFR inhibitor-resistant non-small cell lung cancer (NSCLC). ASCO Meeting Abstract 2014;3215_suppl:8009.

35. Sequist LV, Soria J-C, Gadgeel SM, Wakelee HA, Camidge DR, Varga A, et al. First-in-human evaluation of CO-1686, an irreversible, highly selective tyrosine kinase inhibitor of mutations of EGFR (activating and T790M). ASCO Meeting Abstract 2014;3215_suppl:8010.

36. Sasaki T, Koivunen J, Ogino A, Yanagita M, Nikiforow S, Zheng W, et al. A novel ALK secondary mutation and EGFR signaling cause resistance to ALK kinase inhibitors. Cancer Res. 2011;71:6051-60.

37. Meador CB, Micheel CM, Levy MA, Lovly CM, Horn L, Warner JL, et al. Beyond histology: translating tumor genotypes into clinically effective targeted therapies. Clin Cancer Res. 2014;20:2264-75.

38. Oxnard GR, Binder A, Janne PA. New targetable oncogenes in non-small-cell lung cancer. J Clin Oncol. 2013;31:1097-104.

39. Roberts PJ, Stinchcombe TE. KRAS mutation: Should we test for it, and does it matter. J Clin Oncol. 2013;31:1112-21.

40. Bergethon K, Shaw AT, Ou SH, Katayama R, Lovly CM, McDonald NT, et al. ROS1 rearrangements define a unique molecular class of lung cancers. J Clin Oncol. 2012;30:863-70.

41. Ou S-HI, Bang Y-J, Camidge DR, Riely GJ, Salgia R, Shapiro G, et al. Efficacy and safety of crizotinib in patients with advanced ROS1-rearranged non-small cell lung cancer (NSCLC). ASCO Meeting Abstract 2013;3115_suppl:8032.

42. Paik PK, Arcila ME, Fara M, Sima CS, Miller VA, Kris MG, et al. Clinical characteristics of patients with lung adenocarcinomas harboring BRAF mutations. J Clin Oncol. 2011;29:2046-51.

43. Lipson D, Capelletti M, Yelensky R, Otto G, Parker A, Jarosz $M$, et al. Identification of new ALK and RET gene fusions from colorectal and lung cancer biopsies. Nat Med. 2012;18:382-4.

44. Arcila ME, Chaft JE, Nafa K, Roy-Chowdhuri S, Lau C, Zaidinski $\mathrm{M}$, et al. Prevalence, clinicopathologic associations, and molecular spectrum of ERBB2 (HER2) tyrosine kinase mutations in lung adenocarcinomas. Clin Cancer Res. 2012;18:4910-8.

45. Comprehensive molecular profiling of lung adenocarcinoma. Nature. 2014;511:543-50.

46. Lira ME, Choi YL, Lim SM, Deng S, Huang D, Ozeck M, et al. A single-tube multiplexed assay for detecting ALK, ROS1, and RET fusions in lung cancer. J Mol Diagn. 2014;16:229-43.

47. Travis WD, Brambilla E, Noguchi M, Nicholson AG, Geisinger $\mathrm{K}$, Yatabe $\mathrm{Y}$, et al. Diagnosis of lung cancer in small biopsies and cytology: Implications of the 2011 International Association for the Study of Lung Cancer/American Thoracic Society/European Respiratory Society classification. Arch Pathol Lab Med. 2013;137:668-84.

48. Diaz LA Jr, Bardelli A. Liquid biopsies: Genotyping circulating tumor DNA. J Clin Oncol. 2014;32:579-86.

49. Esposito A, Bardelli A, Criscitiello C, Colombo N, Gelao L, Fumagalli L, et al. Monitoring tumor-derived cell-free DNA in patients with solid tumors: Clinical perspectives and research opportunities. Cancer Treat Rev. 2014;40:648-55.

50. O'Flaherty JD, Gray S, Richard D, Fennell D, O'Leary JJ, Blackhall FH, et al. Circulating tumour cells, their role in metastasis and their clinical utility in lung cancer. Lung Cancer. 2012;76:19-25.

51. Maheswaran S, Sequist LV, Nagrath S, Ulkus L, Brannigan B, Collura CV, et al. Detection of mutations in EGFR in circulating lung-cancer cells. N Engl J Med. 2008;359:366-77.

52. Taniguchi K, Uchida J, Nishino K, Kumagai T, Okuyama T, Okami $\mathrm{J}$, et al. Quantitative detection of EGFR mutations in circulating tumor DNA derived from lung adenocarcinomas. Clin Cancer Res. 2011;17:7808-15. 